\title{
Neural Code: Another breach in the wall?
}

Response to the target article by Romain Brette:

Is coding a relevant metaphor for the brain?

\section{Chloé Huetz, Samira Souffi, Victor Adenis, Jean-Marc Edeline}

NeuroPSI, UMR CNRS 9197, Dept Cognition \& Behavior

Université Paris-Sud, 91405 Orsay, France

chloe.huetz@u-psud.fr

samira.souffi@u-psud.fr

victor.adenis@u-psud.fr

jean-marc.edeline@u-psud.fr

http://neuro-psi.cnrs.fr/

\begin{abstract}
Brette presents arguments that query the existence of the neural code. However he has neglected certain evidence that could be viewed as proof that a neural code operates in the brain. Albeit these proofs show a link between neural activity and cognition, we discuss why they fail to demonstrate the existence of an invariant neural code.
\end{abstract}


By questioning the existence of the neural code, Romain Brette opens again a strong debate between representational views of the brain (cognitivism and computationalism) and sensorimotor/enaction theories (O'Regan and Noë, 2001, Varela et al 1991), his preference being the latter. According to his view, all cognitive functions, particularly action and perception are viewed as means to interact with the world, without the need to build internal representations of it. Neural activity during perception should be viewed as the result of the organism's interaction with the world taking into account all possible influences, such as its internal state and its actions resulting in a given percept. Therefore, as the brain does not manipulate representations, it is senseless to try to decipher any code supposed to encrypt representations in neural activity. The results of three research fields focusing on proving that a particular neural code is at play should be addressed by Brette's review to strengthen his point.

First, in sensory physiology, research on tuning curves has been extended to naturalistic stimuli and is divided into two complementary approaches: encoding and decoding. Based on models of the stimulus-response function, these approaches rely on the idea that neural activity encodes some features of the external world. Successful reconstructions of complex stimuli based on neural responses (decoding), or successful predictions of responses to new stimuli (encoding) are viewed as proofs that the neural code has been cracked. Interpreting these results in light of Brette's arguments seems necessary. Initially, the stimulus reconstruction method (decoding) was performed either with simple artificial stimuli (Bialek et al. 1991) or in peripheral sensory systems (Warland et al 1997, Rieke et al 1995). More recently, studies have reconstructed natural stimuli from cortical responses (Miyawaki et al 2008, Naselaris et al 2009, Akrabi et al 2019), opening the spectacular expectation to read subjects' percepts. In the auditory modality, encoding models were used to investigate neural selectivity to a variety of acoustic properties such as phonetic features (Mesgarani et al, 2014), pitch (Oxenham 2018), timbre and rhythm (Woolley et al 2009). To achieve good performance, the stimulus/response models used in decoding/encoding approaches rely on features such as trial averaging, statistics of natural stimuli, starting time of the stimulus. Thus, the right interpretation should be that an "ideal observer" with an a priori knowledge of the experimental design can infer the stimulus (in the decoding approach) or the neural response (in the encoding approach). Noteworthy, this field has led to an interesting drift from the idea of a fixed relationship between stimulus and 
neural responses to a more dynamic model, and is now tackling the mechanisms by which sensory responses are modulated by learning, context and history (Williamson et al, 2016; Fritz et al 2005; Holdgraf et al. 2016).

Second, the field of neuroprosthetic devices offers demonstrations of causal links between neural code and brain functions. The most successful of these devices, cochlear implant (CI), operates with blunt stimulations of auditory nerve terminals. Despite a large current spread in the tympanic ramp, CI allows implanted subjects to have percepts and recover speech understanding. Even though there are huge differences between normal cochlea and $\mathrm{CI}$, the fact that CIs restore hearing can be viewed as a proof that the neural code at play in the periphery has been deciphered and is successfully implemented in a prosthetic device. However, the CI settings that leads to speech comprehension considerably differ from one subject to another, as do the strategies leading to the largest evoked responses in auditory cortex (Adenis et al 2018). Thus, in contrast with the genetic code that is invariant across cells and species, the neural code (understood as changes in neural activity in adaption to a CI) is probably specific for each individual and/or each type of neuron. In line with sensorimotor theories, success of $\mathrm{CI}$ shows that the brain is using a new input in a way it can interact again with the environment, which might be the basis of hearing restoration.

A third important field investigates the effect of disrupting a particular feature of neural activity on a cognitive skill. In the visual system, disruption of physiological activity in the primate area MT during presentation of moving stimuli bias the perceptive judgment of a behaving animal (Salzman et al 1990; Salzman \& Newsome 1994) thus making the first link between neural code (understood as a pattern of activity of specific neurons) and behavioral performance. More recently, studies performed in the hippocampus have showed that disrupting the replay of spiking patterns occurring across neuronal ensembles during the sharp wave ripples profoundly alters the memory of previously acquired information (Girardeau et al 2009; Ego-Stengel \& Wilson 2010). These data reinforce the notion that neuronal activity patterns do correlate with the acquired information. More importantly, associating a rewarding stimulation of the medial forebrain bundle with an hippocampal place cell activity induced a place preference at the place cell location (Lavilléon et al 2015), demonstrating causal links 
between a particular place cell's firing rate and a specific location memory. In all these examples, the exact neural activity feature (its firing rate or its temporal spike patterns) correlated with the animal's location is unknown, but causal relationships do exist. Yet, causality is not enough to define a neural code.

Clearly, more caution is necessary when discussing the neural code as overstatements made (Ferster \& Spruston 1995; Panzeri et al 2017) tend to generate the illusion that (1) the same code operate in any sensory and motor systems, which is obviously not the case and (2) the brain's cognitive functions consist of manipulating encoded representations of the world, a theory that is controversial. Does this mean that the concept of neural code should be abandoned or should it be used to describe studies linking neural activity to brain function? We believe that the neural code definition should be freed from the notion of representation and we should clarify what we refer to when investigating the neural mechanism of brain functions.

\section{References}

Adenis V, Gourévitch B, Mamelle E, Recugnat M, Stahl P, Gnansia D, Nguyen Y, Edeline JM. (2018) ECAP growth function to increasing pulse amplitude or pulse duration demonstrates large inter-animal variability that is reflected in auditory cortex of the guinea pig. PLoS One. 13(8):e0201771.

Akbari H, Khalighinejad B Herrero JL, Mehta AD, Mesgarani N. (2019) Towards reconstructing intelligible speech from the human auditory cortex. Sci Rep. Jan $29 ; 9(1): 874$.

Bialek W, Rieke F, de Ruyter van Steveninck RR, Warland D. (1991) Reading a neural code. Science 252: 1854-1857.

de Lavilléon G, Lacroix MM, Rondi-Reig L, Benchenane K. (2015) Explicit memory creation during sleep demonstrates a causal role of place cells in navigation. Nat Neurosci. 18 (4):493-5.

Ego-Stengel V, Wilson MA. (2010) Disruption of ripple-associated hippocampal activity during rest impairs spatial learning in the rat. Hippocampus. 20(1):1-10.

Ferster D, Spruston N. (1995) Cracking the neuronal code. Science. 270(5237):756-7.

Girardeau G, Benchenane K, Wiener SI, Buzsáki G, Zugaro MB. (2009) Selective suppression of hippocampal ripples impairs spatial memory. Nat Neurosci. 12(10):1222-3

Fritz J, Elhilali M, Shamma S. (2005) Active listening: task-dependent plasticity of spectrotemporal receptive fields in primary auditory cortex. Hear Res, 206(1-2):159-76. 
Holdgraf CR, de Heer W, Pasley B, Rieger J, Crone N, Lin JJ, Knight RT, Theunissen FE. (2016) Rapid tuning shifts in human auditory cortex enhance speech intelligibility. Nat Commun. 7:13654.

Mesgarani N, Cheung C, Johnson K, Chang EF. (2014) Phonetic feature encoding in human superior temporal gyrus. Science. 343(6174):1006-10.

Miyawaki Y, Uchida H, Yamashita O, Sato MA, Morito Y, Tanabe HC, Sadato N, Kamitani Y. (2008) Visual image reconstruction from human brain activity using a combination of multiscale local image decoders. Neuron. 60(5):915-29.

Naselaris T, Prenger RJ, Kay KN, Oliver M, Gallant JL. (2009) Bayesian reconstruction of natural images from human brain activity. Neuron. 63(6):902-15.

O'Regan J.K., Noë A. (2001) A sensorimotor account of vision and visual consciousness. Behav Brain Sci. Oct;24(5):939-73.

Oxenham AJ. (2018) How we hear: The perception and neural coding of sound. Annu Rev Psychol. 69:27-50

Panzeri S, Harvey CD, Piasini E, Latham PE, Fellin T. (2017) Cracking the Neural Code for Sensory Perception by Combining Statistics, Intervention, and Behavior. Neuron. 93(3):491-507.

Rieke F, Bodnar DA, Bialek W. (1995) Naturalistic stimuli increase the rate and efficiency of information transmission by primary auditory afferents. Proc Biol Sci. 262(1365):259-65.

Salzman CD, Britten KH, Newsome WT. (1990) Cortical microstimulation influences perceptual judgements of motion direction. Nature. 346(6280):174-7.

Salzman CD, Newsome WT. (1994) Neural mechanisms for forming a perceptual decision. Science. 264(5156):231-7.

Varela, F. J., Thompson, E., \& Rosch, E. (1991). The embodied mind: Cognitive science and human experience. Cambridge, MA, US: The MIT Press.

Warland DK, Reinagel P, Meister M. (1997) Decoding visual information from a population of retinal ganglion cells. J Neurophysiol. Nov;78(5):2336-50.

Williamson RS, Ahrens MB, Linden JF, Sahani M. (2016) Input-Specific Gain Modulation by Local Sensory Context Shapes Cortical and Thalamic Responses to Complex Sounds. Neuron. 91(2):467-81.

Woolley SM, Gill PR, Fremouw T, Theunissen FE. (2009) Functional groups in the avian auditory system. J Neurosci. 29(9):2780-93. 